Classification

Physics Abstracts

$61.70 \mathrm{~J}-61.80 \mathrm{~F}-82.50$

\title{
Recombination enhanced dislocation glide in silicon carbide observed in-situ by transmission electron microscopy
}

\author{
Koji Maeda, Kunio Suzuki( $\left.{ }^{\star}\right)$ and Masaki Ichihara( $\left(^{\star}\right)$ \\ Department of Applied Physics, faculty of Engineering, University of Tokyo, Hongo, Tokyo 113, Japan
}

(Received January 18, 1993; accepted March 3, 1993)

\begin{abstract}
The recombination enhanced dislocation glide in a $6 \mathrm{H}-\mathrm{SiC}$ single crystal was studied by in-situ transmission electron microscopy. Electron beam irradiation even at room temperature induced reversible increase in the glide velocity of basal $90^{\circ}$-partial dislocations in proportion to the beam intensity. Evidences for enhancement of the double kink formation as well as for the kink migration are presented.
\end{abstract}

\section{Introduction.}

Electronic excitation of semiconductors such as $\mathrm{Si}$ and GaAs is known to induce remarkable enhancement of dislocation glide [1]. The effect is well interpreted in terms of the recombination enhanced defect reaction (REDR) mechanism [2]. In the REDR mechanism, we suppose that on recombination of an excited minority carrier at an electronic center associated with a defect, the electronic energy is released non-radiatively to induce local vibrations of the defect, which leads to reaction or some rearrangement of the defect itself.

Among many tetragonally coordinated crystals in which this recombination enhanced dislocation glide (REDG) effect has been observed, wide-gap semiconductors ( $\mathrm{GaP}$ [3], ZnS [4], CdS [5]) exhibit generally a strong REDG effect. This is due to the fact that the amount of energy to be released upon carrier recombination tends to increase with the band gap energy [6]. Silicon carbide (SiC) has a wide band-gap energy $\left(E_{g}=2.4 \mathrm{eV}\right.$ to $3.3 \mathrm{eV}$ depending on the polytype $)$ and therefore is expected to be one of such crystals. $\mathrm{SiC}$ is a prospective material for blue light emitting diodes and this fact renders a technological importance to the study of SiC because the REDG effect is known to cause rapid degradation of photonic devices [6].

Pilyankevich and Britun [7] reported that basal dislocations in $\mathrm{SiC}$ of the $6 \mathrm{H}$ polytypic structure $\left(E_{g}=2.8 \mathrm{eV}\right)$ glide even at room temperature while the crystal is examined in a transmission electron microscope (TEM). This fact, presumably a manifestation of the REDG effect, is very surprising because $\mathrm{SiC}$ is an extremely hard solid as used for grinding powder and in fact the

$\left(^{\star}\right)$ Institute for Solid State Physics, University of Tokyo, Roppongi, Tokyo 106, Japan. 
mobility of dislocations in $6 \mathrm{H}-\mathrm{SiC}$ is quite low at ambient temperatures [8]. More recently Pirouz and his coworkers reproduced this anomalous effect [9]. The present report describes our most recent work on in-situ TEM observations of dislocation glide in a $6 \mathrm{H}-\mathrm{SiC}$ single crystal.

\section{Experimental procedure.}

The specimens were prepared from a single crystal of $6 \mathrm{H}-\mathrm{SiC}$ grown by a modified Acheson method. After shaped to rectangles of $2 \times 3 \times 6 \mathrm{~mm}^{3}$ in size by a diamond wheel cutter, the crystal was subjected to uniaxial compression at $1560^{\circ} \mathrm{C}$ in a single slip orientation to activate the basal slip preferentially. For the detail of deformation procedure, the readers are referred to our previous work [10]. The deformed crystal was sliced to thin plates along the basal planes, then mechanically polished with diamond abrasives and finally thinned by $\mathrm{Ar}$ ion etching.

TEM observations were conducted in Hitachi H-9000 operated at 100 or $150 \mathrm{kV}$. It should be pointed out that similar experiments which was tried by using a high voltage electron microscope (JEOL, JEM-1250) operated at $1000 \mathrm{keV}$ was unsuccessful presumably because radiation damage, although undetected in TEM images, pinned down dislocations. The basal dislocations observed were all dissociated to Shockley partials in wide separations due to the extremely low stracking fault energy [8]. Hence the partial dislocations were easily resolved by the standard bright-field imaging, though in some cases the weak beam technique was used to investigate the dislocation morphology in more detail. No intentional load was applied on specimens. Dislocation motion at room temperature was recorded by a standard video system.

\section{Dislocation motion under an electron beam.}

Figure 1 shows a sequence of partial dislocation motion recorded in a weak-beam imaging condition. When the electron beam, typically of the order of $10^{4} \AA / \mathrm{m}^{2}$ in current density, was further focussed on such partials, they exhibited instantaneous increase in the glide velocity. Although in this specific example in figure 1 the dislocation motion appears to be somewhat uneven, the average velocity of whole segments and of long-range motion is generally more or less uniform. Figure 2 presents a typical example demonstrating the enhancement effect induced by electron beam. The traveling distance in the ordinate is the distance which was traveled by a straight segment of a single $90^{\circ}$-partial dislocation (images are not shown here) from a starting time to the time indicated in the abscissa. The numerals beside the plot indicate the beam intensities in unit of $\mathrm{A} / \mathrm{m}^{2}$ that were used for the TEM observations. As evident from the figure, the effect of beam illumination is almost completely reversible.

Since the driving force relied on uncontrollable residual stresses which were apt to vary from place to place, the intensity dependence could be examined only by assessing at the same position the relative change of dislocation velocities induced by alteration of the beam intensity. Figure 3 shows the relation between the ratio of the beam intensity and the corresponding relative change in dislocation velocity. The considerable scatter of the experimental data is partly due to the difficulty in precise control of the beam intensity. The solid line indicates the linear dependence of dislocation velocity on the beam intensity, which is one of the features that the REDG effect should exhibit [1].

The magnitude of the REDG effect was found to depend significantly on the type of the dislocations characterized by the sign and the orientation of the dislocation line with respect to the Burgers vector. Figure 4 presents a typical example: The segment marked $D_{1}$ glided under electronic excitation but meanwhile the adjacent segments $D_{2}$ and $D_{3}$ did not show significant movement. A 


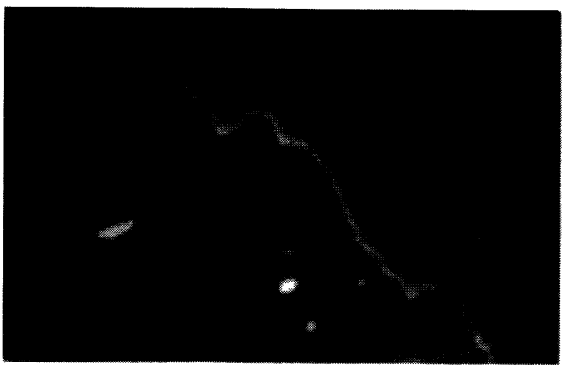

$0 \mathrm{~s}$

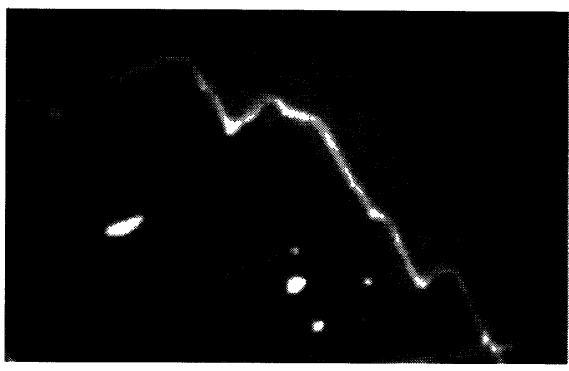

$4 \mathrm{~s}$

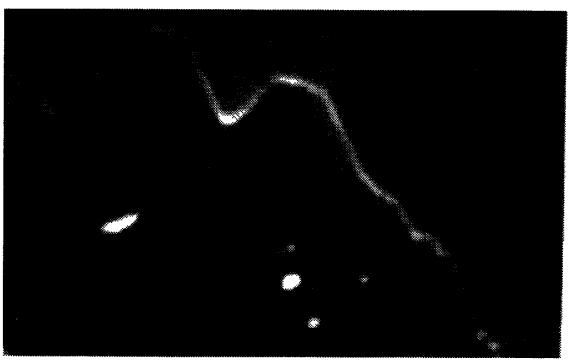

$8 \mathrm{~s}$

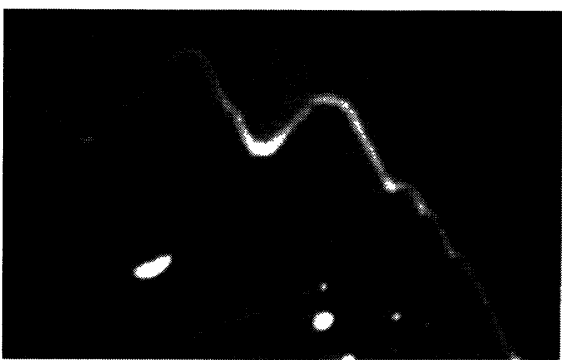

$14 \mathrm{~s}$

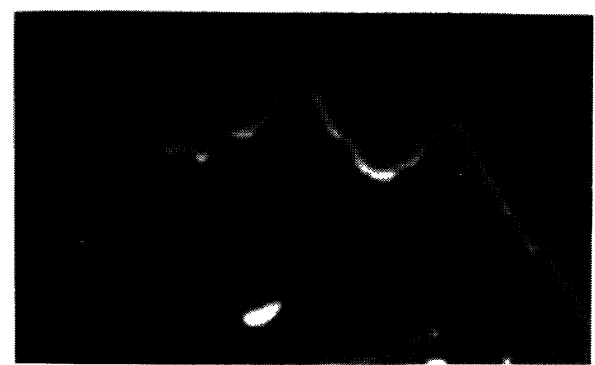

$23 \mathrm{~s}$

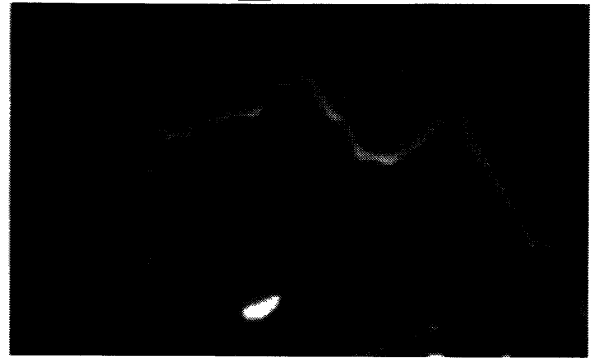

$27 \mathrm{~s}$

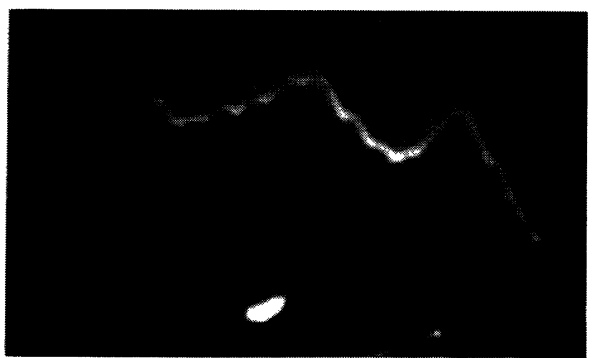

$33 \mathrm{~s}$

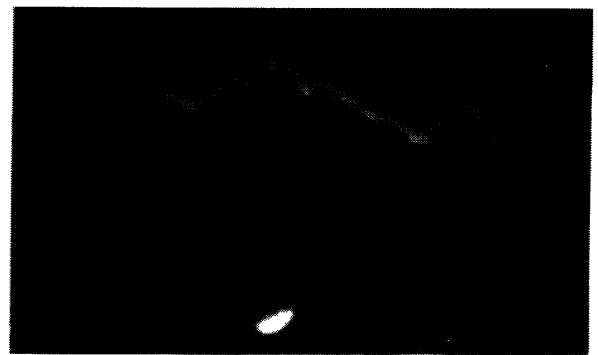

$71 \mathrm{~s}$

$0.1 \mu \mathrm{m}$

Fig. 1. - A sequence of TV recorded weak-beam images showing motion of a basal dislocation in $6 \mathrm{H}-\mathrm{SiC}$ that is enhanced at room temperature by irradiation of $150 \mathrm{kV}$ electron beam 


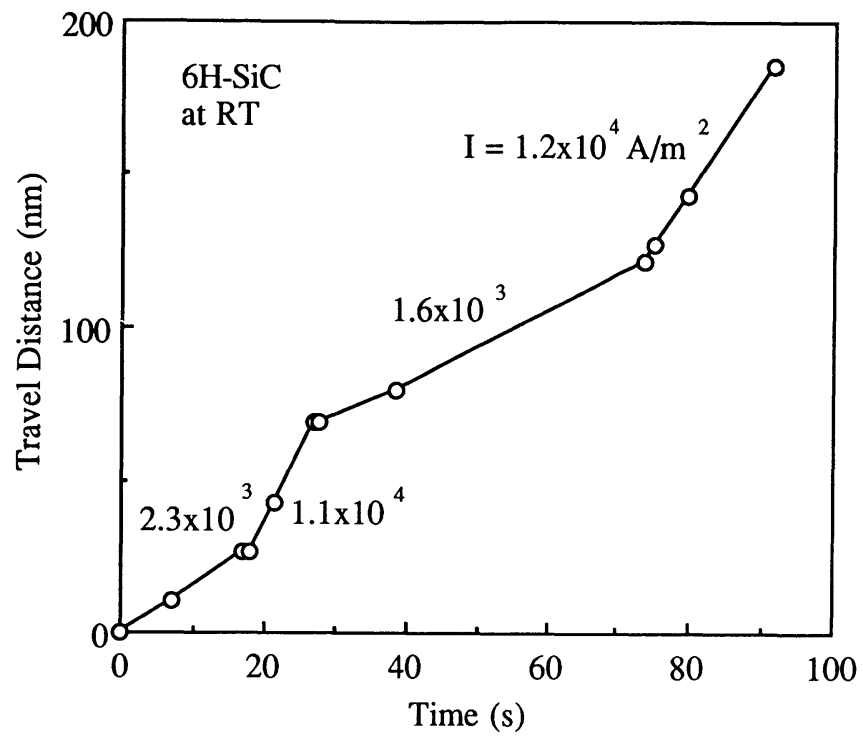

Fig. 2. - Progress of a dislocation with time under different beam intensities in unit of $A / \mathrm{m}^{2}$. Note that the irradiation effect is reversible. The parameter $I$ is not the incident intensity but the one calculated from the beam current penetrating the specimen.

diffraction analysis revealed that the Burgers vector $\mathbf{b}$ was as shown in the figure. The enhanced segments were thus $90^{\circ}$ partials, although the polarity of the core $(\alpha$ or $\beta)$ is not known. As a consequence of this anisotropy in the enhanced mobility of dislocations, the dislocations tended to change their shape to undulating lines with many triangular cusps as found in the previous work by Pilyankevich and Britun [7]. Quite a similar transformation of dislocation segments to saw-tooth shape on electronic excitation was reported for CdTe by Levade and his coworkers [11].

The length of the dislocation segment $D_{1}$ in figure 4 decreased continuously as it advanced with time. Therefore, provided that the stress remained constant during the advancement of the segment, one can extract from its motion the dependence of the dislocation velocity on the dislocation line length. Figure 5 plots the dislocation velocity of the segment $D_{1}$ in figure 4 as a function of the segment length. Except for extremely short segments, the dislocation velocity was almost independent of the dislocation length with a discernible undulation in the plot.

The enhanced dislocation motion was observed in bowing-out segments as shown in figure 6. If one considers that double kink formation is necessary for dislocations to bow-out from the Peierls troughs, this fact implies that it is the double kink formation that is enhanced by electronic excitation. The enhanced motion was observed also in such dislocations rotating from the Peierls trough as shown in figure 7 . Since the slanting dislocations should be composed of a high density of geometrical kinks, this fact suggests that enhancement is taking place in the kink migration as well. This is in common with a similar behavior of dislocations in GaAs observed in in-situ TEM experiments previously reported by Küsters et al. [12].

It has been reported in the literature [11-13] that dislocations in semiconducting crystals that are not stressed intentionally sometimes exhibit vibrational motion between pinning points under an electron beam. A similar vibration of dislocation segments, though not drastic as in the previous cases, was occasionally observed in the present study as well. 


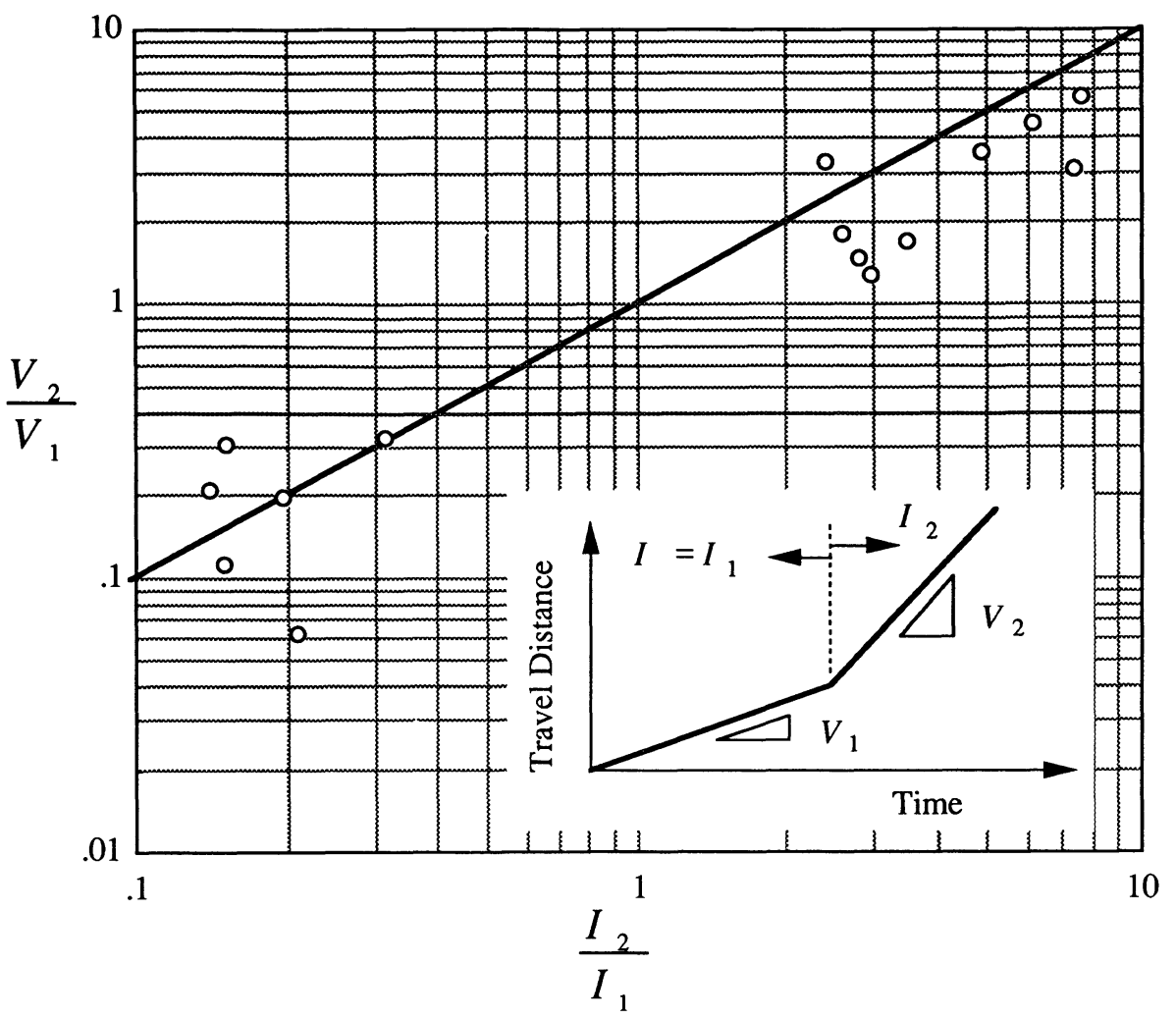

Fig. 3. - Relative change in the dislocation velocity with respect to the change in beam intensity. The solid line indicates the linear dependence.

\section{Discussion.}

According to the kink diffusion model by Hirth and Lothe [14], the rate controlling process of dislocation motion in semiconductors differs in two regimes depending on the dislocation length. If a dislocation has a finite length $L$, such that kink-pairs expand only to the length $L$ without colliding with other kinks generated on the same segment, the velocity of the dislocation should linearly increase with the segment length $L$. If a dislocation is long enough for kink collision to take place, the dislocation velocity should become independent of the dislocation length. The length-independent velocity observed in figure suggests that the dislocation motion, enhanced by electron beam excitation, is in the kink collision regime. This is in contrast to the case of the REDG effect in $\mathrm{ZnS}[4,15]$ where the enhanced dislocation velocity exhibited a linear dependence on the dislocation length.

The fact that the enhancement is reversible and linearly dependent on the irradiation intensity evidences that the present effect is in fact the REDG effect. There are two microscopic processes that possibly be enhanced by electronic excitation, i.e., the (smallest) double kink formation and the kink migration. A theoretical analysis [16] showed that the linear dependence on excitation intensity is predicted only in limited cases: In the kink-collision regime, the linear dependence is obtained only when both the double kink formation and the kink migration are enhanced; in the kink-collisionless regime, enhancement of the double kink formation alone as well as enhance- 


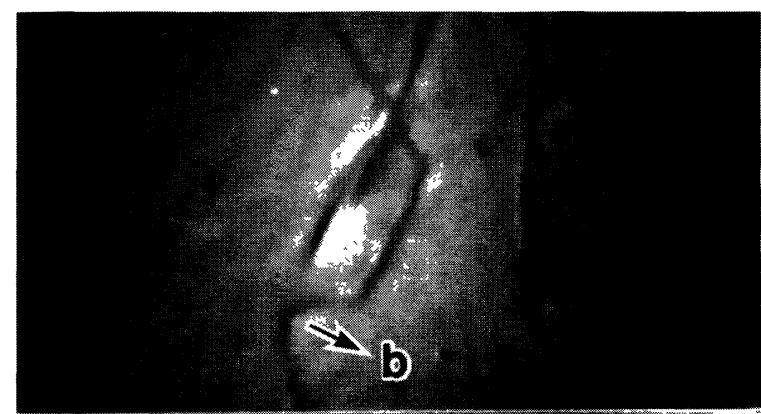

\section{Os}

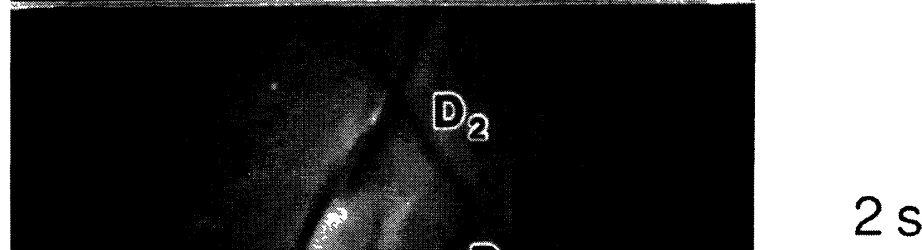

D) 8

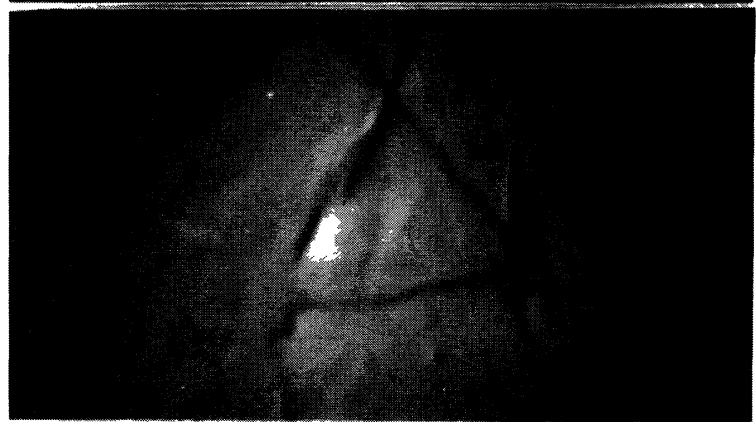

$4 s$

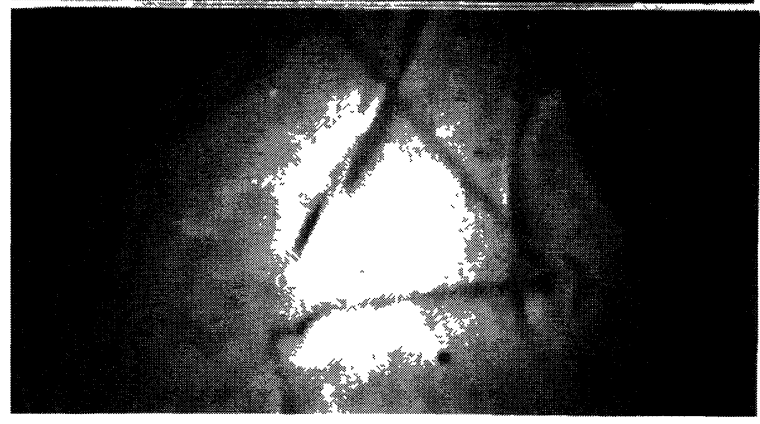

$2 \mathrm{~s}$
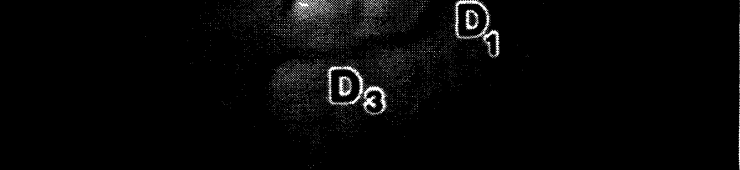

\section{$11 \mathrm{~s}$}

$0.1 \mu \mathrm{m}$

Fig. 4. - Glide enhancement depending on the dislocation type characterized by the Burgers vector $\mathbf{b}$. This also demonstrates the variation of the dislocation velocity with the dislocation line length. 


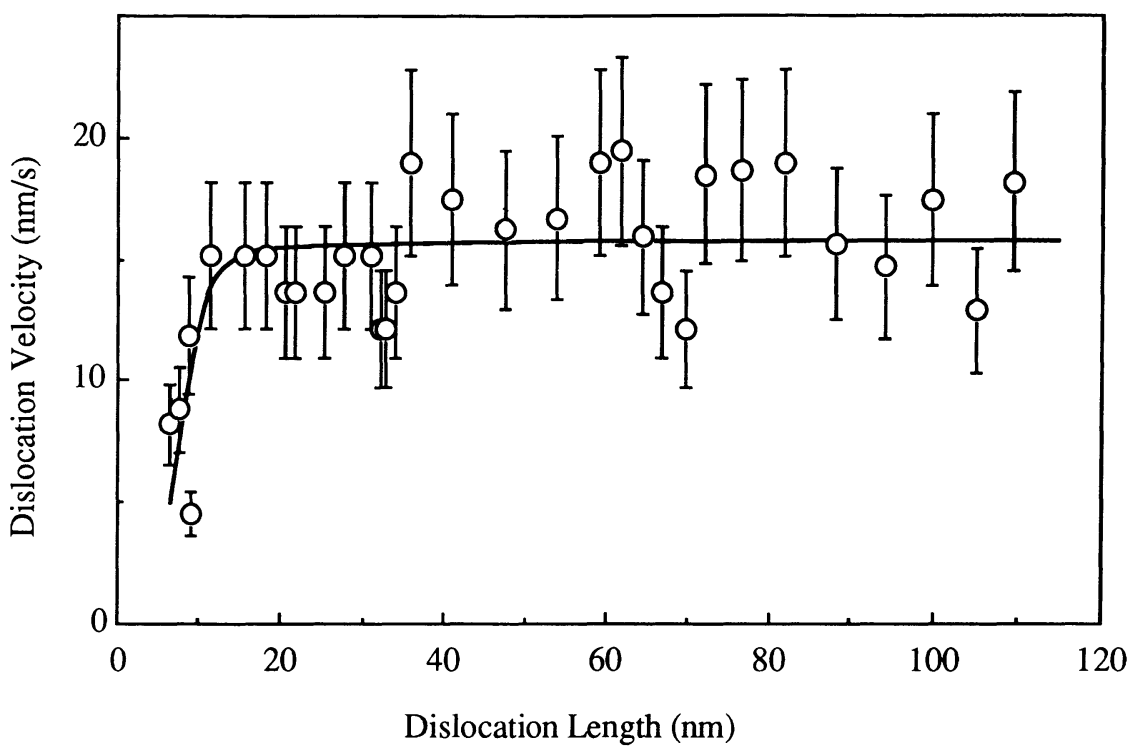

Fig. 5. - The relation between the enhanced velocity of a $90^{\circ}$-partial dislocation (the segment $D_{1}$ in Fig. 4) and the segment length.

ment of the double kink formation and the kink migration can produce the linear dependence. It is important to point out that in any case enhancement of the double kink formation is a requisite for the REDG effect to be linear. The present observations that dislocations bow out from the Peierls trough is consistent with the view that the double kink formation is enhanced.

In principle the kink-collision regime is realized also when the double kink formation is so frequent that multiple kink-pairs are always present on a single line segment. Although a recent study by one of the present author (KM) [17] showed that the length-independent velocity is not necessarily a consequence of kink collision, the length-independent velocity in the present study tempts us to suppose that the enhanced dislocation motion is in the kink-collision regime, because enhancement of double kink formation should result in decrease in the mean separation of kinks. This view may be supported by the appreciably wavy morphology of the dislocation line, as seen in figure 4, that is expected from the presence of kinks in high density. If the dislocation motion is really in the kink-collision regime, we have to conclude from the argument above that the enhancement takes place in the kink migration as well. We have seen already in figure 7 that enhancement of kink migration is inferred also from the TEM observations.

The undulation in the dislocation velocity discernible in figure 5 may be related to the vibrational motion occasionally observed under the electron beam. The vibrational motion of dislocations in CdS was explained by Lu and Cockayne in terms of the interaction between the dislocation line tension and the electrostatic repulsion of a kink on a charged dislocation line, the last of which is often supposed in semiconductors. However, the fact that dislocations in metallic alloys also sometimes exhibit similar vibrations on specimen heating [18] seems to rule out the mechanism that is peculiar to semiconductors. The vibration may be caused by an alternate change in the internal stress induced by rearrangement of surrounding dislocations that become mobile on heating or by electronic excitation. The enhanced double kink formation, by either heating or electronic excitation, solely may be responsible for the vibration. Elucidation of the exact mechanism is a subject of the future study. 


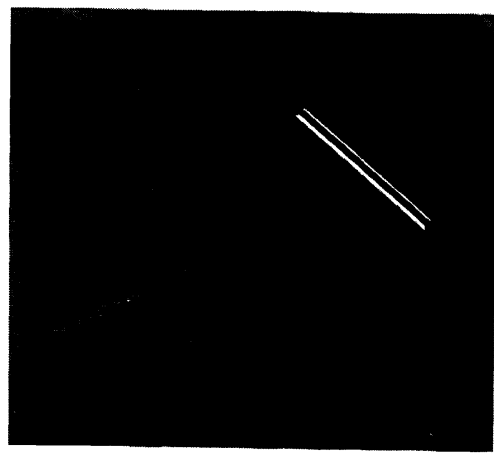

\section{$0 \mathrm{~s}$}

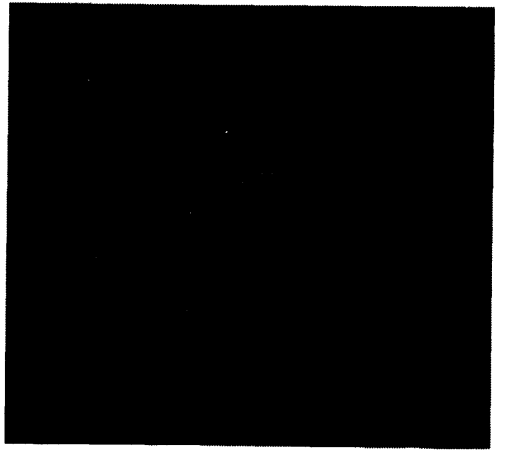

$6 s$

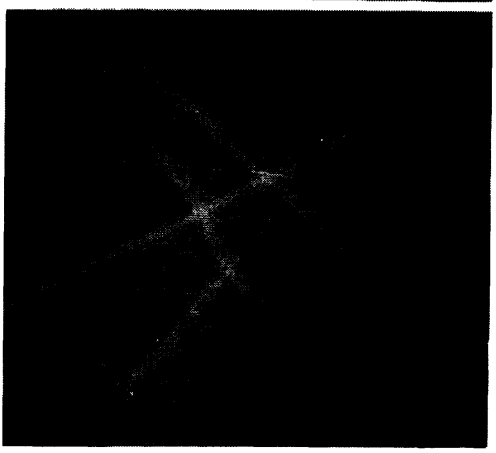

$25 \mathrm{~s}$

Fig. 6. - Dislocation bowing-out from the Peierls trough showing that the double kink formation is enhanced. The direction of the Peierls trough is indicated by a straight line in the top frame.

\section{Acknowledgements.}

The authors are grateful to Taiheiyo Rundum Corporation for supplying the $6 \mathrm{H}$-SiC single crystals. They are indebted to Dr.S. Fujita for sample preparation and to Mr.T. Hashimoto for help in deforming SiC crystals. The authors also wish to thank Prof. P. Pirouz, the Case Western Reserve University, for his private communication on his TEM study and Prof. S. Takeuchi, the Institute for Solid State Physics, for his encouragement and affording facilities for high temperature compression. A part of this work has been supported by Grant-in-Aid for Scientific research from the Ministry of Education, Science an Culture of Japan and the Nippon Glass Foundation for Materials Science. 


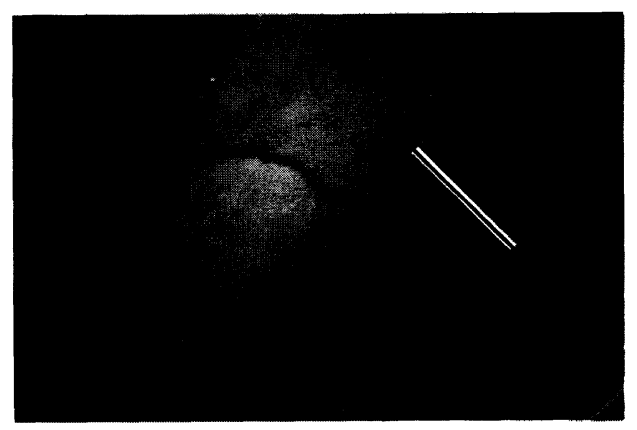

Os
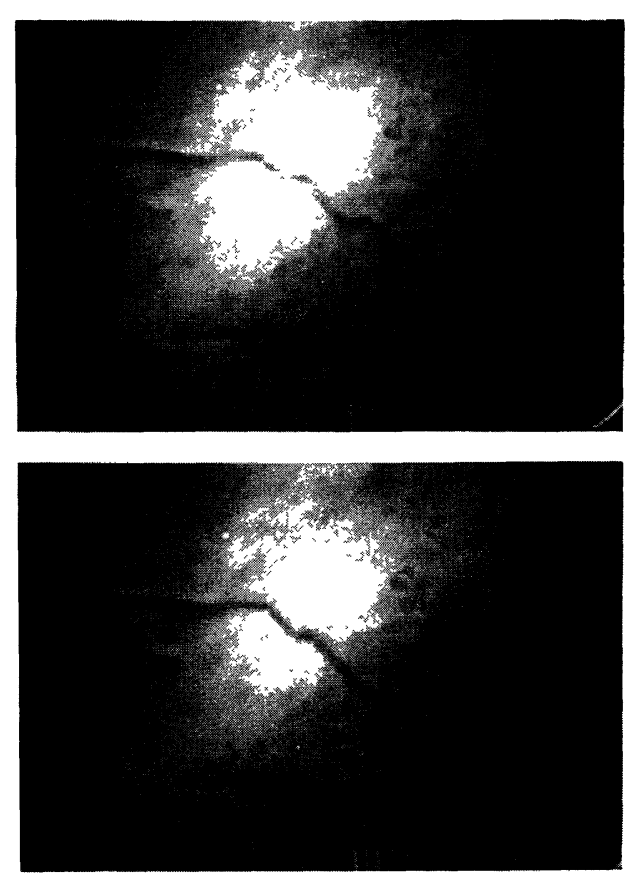

$5 s$

$0.1 \mu \mathrm{m}$

Fig. 7. - Successive motion of a dislocation initially slanting from the Peierls trough in a [110] direction (indicated in the top frame with a straight line), and eventually straightening along the potential trough, which suggests that enhancement takes place in the kink migration as well.

\section{References}

[1] Maeda K., Sato M., Kubo A. and Takeuchi S., J. Appl. Phys. 54 (1983) 161.

[2] Weeks J.D., Tully J.C., Kimerling L.C., Phys. Rev. B 12 (1975) 3286.

[3] Maeda N. and TakeuChi S., Japn. J. Appl. Phys. 29 (1990) 1151.

[4] Levade C., CouderC J.J., CAIllard D. and Couret A., Electron Microsc. Plasticity a Fracture Research Mater. Eds. U. Messerschmidt, F. Appel, J. Heydenreich and V. Schmidt (akademie Verlag, Berlin, 1989) p. 199.

[5] NegRiI V.D., Osipyan Yu.A. and LomaK N.V., Phys. Stat. Sol. (a), 126 (1991) 49.

[6] Maeda K., Yamashita Y., Maeda N. and TaKeUCHI S., Mat. Res. Soc. Symp. Proc. Vol. 184 (1990) 69.

[7] PilyankeVich A.N. and Britun V.F., Phys. Stat. Sol. (a), 82 (1984) 449.

[8] MAeda K., SuZuki K., Fujita S., IChihara M. and Hyodo S., Philos. Mag. A 57 (1988) 573.

[9] PIROUZ P., private communication. 
[10] Fujita S., Maeda K. and Hyodo S., Philos. Mag. A 55 (1987) 203.

[11] Levade C., Couderc J.J., Vanderschaeve G., Caillard D. and Couret A., Appl. Surf. Sci 50 (1991) 119.

[12] Küsters K.-H., De Cooman B.C. and CarTer C.B., Philos. Mag. A 53 (1986) 141.

[13] LU G. and CocKayne D.J., Philos. Mag. A 53 (1986) 297.

[14] HiRTH J.P. and LOTHE J., Theory of Dislocations, (McGraw-Hill, New York, 1968) p.484.

[15] VanderschaeVe G., LeVade C., FAREss A., Couderc J.J. and CAillard D., J. de Phys. IV. C6 (1991) 305.

[16] MAEdA N. and Takeuchi S., Inst. Phys. Conf. Ser. No. 104 (1989) 303.

[17] Yamashita Y., MaEda K., Fujita K., Usami N., SuZuki K., FuKatsu S., Mera Y. and Shiraki Y., Philos. Mag. Lett. 67 (1993) 165.

[18] SAKA H., private communication. 